\title{
A IDENTIDADE DO CUIDADO DE ENFERMAGEM NA PRIMEIRA DÉCADA DO SÉCULO XXI*
}

Dâmarys Kohlbeck de Melo Neu Ribeiro' ${ }^{1}$ Eliane Cristina Sanches Maziero² ${ }^{2}$ Juliana Taques Pessoa da Silveira³, Susanne Elero Betiolli ${ }^{4}$, Nen Nalú Alves das Mercês ${ }^{5}$

\begin{abstract}
RESUMO: Este estudo objetivou buscar evidências científicas acerca da identidade do cuidado de enfermagem na prática profissional na primeira década do século XXI. Trata-se de revisão integrativa na qual foram analisados os resumos de 335 artigos científicos. Prevaleceram artigos de pesquisa qualitativa ( $\mathrm{n}=151 ; 45,07 \%)$, realizadas no âmbito hospitalar ( $\mathrm{n}=176 ; 52,5 \%)$, na dimensão do cuidado ( $\mathrm{n}=198 ; 59,2 \%)$ e na temática saúde do adulto (n=107;31,94\%). Os atributos do cuidado identificados foram agrupados em três categorias: Importância do conhecimento para a efetivação do cuidado; Sistematização do cuidado; e Cuidado humanizado, pautado nas relações e interações. Na primeira década do século XXI a identidade do cuidado de enfermagem esteve pautada no conhecimento do profissional enfermeiro, na sistematização da assistência de enfermagem e na humanização do cuidado de enfermagem.
\end{abstract}

DESCRITORES: Enfermagem; Cuidados de enfermagem; Papel do profissional de enfermagem.

\section{THE IDENTITY OF NURSING CARE IN THE FIRST DECADE OF THE XXI CENTURY*}

ABSTRACT: This study aimed to seek scientific evidence regarding the identity of nursing care in professional practice in the first decade of the XXI century. This is an integrative review in which were analyzed the abstracts of 335 scientific articles. There was a prevalence of qualitative research articles $(\mathrm{n}=151 ; 45.07 \%)$, undertaken in the hospital setting $(\mathrm{n}=176 ; 52.5 \%)$, in the dimension of the care $(\mathrm{n}=198 ; 59.2 \%)$ and in the topic of adult's health $(\mathrm{n}=107 ; 31.94 \%)$. The attributes of the care identified were grouped into three categories: Importance of the knowledge for putting the care into effect; Systematization of the care; and Humanized care based on the relations and interactions. In the first decade of the XXI century, the identity of the nursing care was based on the knowledge of the professional nurse, on the systematization of the nursing assistance, and on the humanization of the nursing care. DESCRIPTORS: Nursing; Nursing care; Role of the nursing professional.

\section{LA IDENTIDAD DEL CUIDADO DE ENFERMERÍA EN LA PRIMERA DÉCADA DEL SIGLO XXI*}

RESUMEN: Este estudio tuvo el objetivo de buscar evidencias científicas acerca de la identidad del cuidado de enfermería en la práctica profesional en la primera década del siglo XXI. Es una revisión integrativa en la cual fueron analizados los resúmenes de 335 artículos científicos. Predominaron artículos de investigación cualitativa ( $\mathrm{n}=151 ; 45,07 \%)$, realizadas en el ámbito hospitalar $(\mathrm{n}=176 ; 52,5 \%)$, en la dimensión del cuidado ( $\mathrm{n}=198 ; 59,2 \%$ ) y en la temática salud del adulto $(\mathrm{n}=107 ; 31,94 \%)$. Los atributos del cuidado identificados fueron reunidos en tres categorías: Importancia del conocimiento para efectivar el cuidado; Sistematización del cuidado; y Cuidado humanizado, basado en las relaciones e interacciones. En la primera década del siglo XXI, la identidad del cuidado de enfermería estuvo basada en el conocimiento del profesional enfermero, en la sistematización de la asistencia de enfermería y en la humanización del cuidado de enfermería. DESCRIPTORES: Enfermería; Cuidados de enfermería; Papel del profesional de enfermería.

\footnotetext{
*Artigo oriundo da disciplina Enfermagem e a Prática Profissional do curso de Mestrado Acadêmico do Programa de Pós-Graduação em Enfermagem da Universidade Federal do Paraná - PPGENF UFPR.

${ }^{1}$ Enfermeira. Mestre em Enfermagem. Doutoranda em Enfermagem pelo PPGENF UFPR. Bolsista CAPES. Membro do Grupo Multiprofissional de Pesquisa sobre Idosos - GMPI.

${ }^{2}$ Enfermeira do Hospital Infantil Waldemar Monastier. Mestre em Enfermagem. Membro do Grupo de Estudos Multiprofissional em Saúde do Adulto - GEMSA.

${ }^{3}$ Enfermeira. Mestre em Enfermagem. Membro do Núcleo de Estudos, Pesquisa, Extensão em Cuidado Humano em Enfermagem NEPECHE.

${ }^{4}$ Enfermeira. Mestre em Enfermagem. Doutoranda em Enfermagem pelo PPGENF UFPR. Bolsista CAPES. Membro do GMPI.

${ }^{5}$ Enfermeira. Doutora em Enfermagem. Professora do Departamento de Enfermagem e do PPGENF UFPR. Membro do NEPECHE.
}

Autor correspondente:

Recebido: $22 / 08 / 2012$

Eliane Cristina Sanches Maziero

Aprovado: 20/11/2012

Hospital Infantil Waldemar Monastier

Rua XV de novembro, 3701 - 83601-030 - Campo Largo-PR-Brasil

E-mail: elicris_maziero@yahoo.com.br 


\section{INTRODUÇÃO}

O ser humano é complexo, dotado de necessidades a serem atendidas, como as fisiológicas, de segurança, relacionamento, autoestima, e realização pessoal. De forma, a suprir algumas dessas necessidades, principalmente no que concerne à atenção à saúde, é comum que as pessoas busquem por profissionais especializados, e se deparem com encontros terapêuticos, sistemáticos e, por muitas vezes, impessoais. Ao transpor essa barreira, todo profissional de saúde cria condições de oferecer uma relação de cuidado.

O cuidado, do latim cura e do alemão sorge, na perspectiva ontológica existencial de Heidegger, se refere às relações entre o modo de ser dos humanos; os modos de compreenderem a si mesmos e ao seu mundo; e seus modos de agir e interagir. $\mathrm{O}$ cuidado na concepção Heideggeriana tem duas dimensões: sorge, significando angústia e fürsorge, solicitude $^{(1)}$. Para tanto, Heidegger designa cuidado como o próprio ser do ser humano (ser-aí), o ser-no-mundo. Em outras palavras, compreende todas as possibilidades da existência que estejam vinculadas às coisas e aos outros homens, além de denominadas pela situação ${ }^{(2-3)}$.

Embora o cuidado, na filosofia Heideggeriana, não diga respeito ao cuidar ou descuidar no sentido operativo do senso comum, mas ao movimento do existir, na abertura do ser, do ente, o cuidado constitui na essência da existência humana ${ }^{(1)}$.

Pode-se compartilhar também a concepção de cuidado de Ayres, que considera cuidado como

designação de uma atenção à saúde imediatamente interessada no sentido existencial da experiência do adoecimento, físico ou mental, e, por conseguinte, também das práticas de promoção, proteção e recuperação da saúde ${ }^{(2: 22)}$.

Em relação à enfermagem, a partir de pesquisa documental, alguns autores apresentaram à comunidade científica o modo como a profissão foi registrada nas telas de pintores do século XVI ao século $\mathrm{XX}$, relacionando os conceitos expressos com a história da enfermagem. O cuidado é apontado como uma constante, a essência da enfermagem, desde o século XVI até o século passado ${ }^{(4)}$.

Partindo da concepção de cuidado citada por Ayres $^{(2)}$, e relacionando-a à enfermagem, objetivou-se no presente trabalho buscar evidências científicas acerca da identidade do cuidado de enfermagem na prática profissional na primeira década do século XXI. O termo identidade tem origem do latim identitas, cujo conceito possui três definições. A primeira é de Aristóteles, que a define como unidade de substância, e ainda aponta que as coisas só são idênticas se é idêntica a definição da substância delas. A unidade da substância (definição que a expressa) é, desse ponto de vista, o significado da identidade. Essa definição foi adotada por Hegel, que posteriormente definiu a essência como "identidade consigo mesma"(3:612).

Leibniz formulou a segunda definição, que aproxima o conceito de identidade ao de igualdade. Definição análoga foi desenvolvida por Wolff que definiu como idênticas as coisas que se podem substituir uma à outra, salvaguardando quaisquer de seus predicados. A terceira concepção foi defendida por Waismann, na qual identidade pode ser estabelecida ou reconhecida com base em qualquer

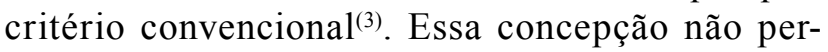
mite estabelecer um definitivo significado para o termo identidade ou o critério para reconhecê-la, no entanto, dentro de determinado sistema linguístico, é possível determinar esse critério de forma convencional e oportuna.

Quanto ao cuidado na prática profissional de enfermagem, existem diferentes definições, porém, ressalta-se a ausência de publicações voltadas à identidade do cuidado de enfermagem. Diante do exposto, o presente estudo teve por objetivo buscar evidências científicas acerca da identidade do cuidado de enfermagem na prática profissional na primeira década do século XXI.

\section{MÉTODO}

Trata-se de uma revisão integrativa da produção científica brasileira sobre a identidade do cuidado de enfermagem na prática profissional na primeira década do século XXI. Para estruturar esta revisão utilizaram-se as seguintes etapas metodológicas ${ }^{(5)}$ : seleção da questão para revisão; 2) estabelecimento de critérios para seleção da amostra; 3) apresentação das características da pesquisa primária; 4) análise dos dados; 5) interpretação dos resultados; e 6) apresentação da revisão.

A questão norteadora da revisão integrativa foi: qual a identidade do cuidado de enfermagem na prá- 
tica profissional da primeira década do século XXI?

Os critérios para seleção da amostra foram: a) ser artigo científico publicado nacional ou internacionalmente, originado no Brasil; b) ter sido publicado na primeira década do século XXI; c) estar disponível online para consulta, em periódicos indexados nas bases de dados eletrônicas consultadas.

Foi realizada busca das produções na Biblioteca Virtual de Saúde (BVS) a partir das bases de dados Literatura Latino-americana e do Caribe em Ciências da Saúde (LILACS), Literatura Internacional em Ciências da Saúde (MEDLINE), Base de Dados da Enfermagem (BDENF) e Scientific Eletronic Library Online (SciELO). Foram utilizados os descritores enfermagem e cuidados de enfermagem.

Os dados foram coletados entre maio e junho de 2011 e a busca inicial resultou em 518 artigos científicos. Na base de dados LILACS foram encontradas 74 publicações, das quais 60 foram excluídas por repetição nas outras bases de dados; duas por não estarem disponíveis online; e 7 que não apresentaram o descritor cuidados de enfermagem, o que totalizou cinco artigos a serem analisados. $\mathrm{Na}$ BDENF foram encontrados 65 artigos, dos quais três foram excluídos por repetição na própria base de dados; 18 artigos por não estarem disponíveis online; e 21 por não apresentarem o descritor cuidados de enfermagem; restando 23 artigos para a análise.

$\mathrm{Na}$ base SciELO os descritores foram inseridos no campo assunto, e encontrou-se 373 artigos científicos. Excluíram-se os que estavam em língua estrangeira $(n=56)$, e 11 por repetição, restando 306 artigos para a análise. Aos descritores eleitos para o estudo, na busca na base de dados MEDLINE, acrescentou-se o país de publicação (Brasil). Resultaram seis artigos, dos quais um era repetido na base BDENF; um não estava disponível online; e três não apresentaram o descritor cuidados de enfermagem. Assim, apenas um artigo foi analisado nesta base de dados.

Portanto, após aplicação dos critérios de inclusão foram excluídos 75 artigos científicos por repetição; 21 por não estarem disponíveis online; 56 por serem em outro idioma; e 31 por não apresentarem o descritor cuidados de enfermagem. Desta forma, a amostra desta revisão foi composta por 335 artigos.

Foi realizada a leitura dos resumos e as informações foram coletadas por meio de um instrumento elaborado pelas autoras, que contemplou os seguintes itens: base de dados, autor(es), periódico, referência, título, resumo, contexto (hospitalar, ensino, atenção primária, entre outros), temática do estudo (saúde coletiva, da criança e adolescente, da mulher, do adulto, do idoso, entre outras), tipo de publicação (pesquisa, reflexão, relato de experiência, revisão ou estudo de caso). Nos estudos caracterizados como pesquisa, foi registrado o tipo de pesquisa (avaliativa, estudo de caso, metodológica, qualitativa, quantitativa, quali-quantitativa), dimensão da prática abordada, atributos do cuidado e considerações finais dos autores.

\section{RESULTADOS}

Os resultados foram apresentados em consonância com a quarta etapa, que consiste na análise dos dados. Da totalidade da amostra $(n=335), 5(1,5 \%)$ artigos foram encontrados na base de dados LILACS, 306(91,35\%) na SciELO, 1(0,30\%) na MEDLINE e $23(6,85 \%)$ na BDENF. Apresentam-se na tabela 1 os tipos de publicação das produções analisadas.

Quanto ao contexto em que os estudos foram realizados, destaca-se o âmbito hospitalar, em $178(53,2 \%)$ publicações. Os que não evidenciaram o contexto no resumo correspondem a $83(24,8 \%)$ artigos. Observou-se 23(6,8\%) publicações na atenção primária; $17(5 \%)$ em nível ambulatorial; $15(4,5 \%)$ publicações no âmbito da academia/ ensino; $12(3,6 \%)$ na educação em saúde; 5(1,5\%) em contexto domiciliar; $1(0,3 \%)$ publicação no contexto empresarial e $1(0,3 \%)$ em Instituição de Longa Permanência para Idosos.

Com relação à dimensão da prática profissional prevaleceu a dimensão Cuidado em 199(59,4\%) publicações, seguida da dimensão Pesquisa observada em 63(18,8\%) publicações e Gerência em 39 $(11,6 \%)$. A dimensão Ensino esteve presente em 29 $(8,7 \%)$ publicações e a dimensão Política em apenas $5(1,5 \%)$. Observa-se no gráfico 1 as temáticas abordadas nos estudos analisados.

Os atributos do cuidado de enfermagem identificados estão relacionados na Tabela 2. A identificação desses atributos possibilitou o agrupamento em três categorias: Importância do conhecimento para a efetivação do cuidado; Sistematização do cuidado; e Cuidado humanizado, pautado nas relações e interações (Tabela 2). As particularidades de cada grupo de publicações serão discutidas na seção seguinte, de maneira a verificar qual a identidade do cuidado na prática profissional nesse início do século XXI. 
Tabela 1 - Tipos de publicações sobre o cuidado de enfermagem por base de dados. Curitiba, 2011

\begin{tabular}{|c|c|c|c|c|c|c|}
\hline \multirow{2}{*}{$\begin{array}{l}\text { Tipo de } \\
\text { publicação }\end{array}$} & \multicolumn{4}{|c|}{ BASE DE DADOS } & \multicolumn{2}{|c|}{ Total } \\
\hline & BDENF & LILACS & MEDLINE & SciELO & $\mathbf{n}$ & $\%$ \\
\hline Estudo de caso & 2 & - & - & 4 & 6 & 1,79 \\
\hline \multicolumn{7}{|l|}{ Pesquisa } \\
\hline Avaliativa & - & - & - & 2 & 2 & 0,60 \\
\hline Estudo de caso & - & - & - & 1 & 1 & 0,30 \\
\hline Metodológica & - & - & - & 2 & 2 & 0,60 \\
\hline Quali-Quanti & - & - & - & 2 & 2 & 0,60 \\
\hline Quantitativa & 2 & - & - & 68 & 70 & 20,90 \\
\hline Qualitativa & 11 & 2 & - & 138 & 151 & 45,07 \\
\hline Reflexão & 4 & 1 & - & 39 & 44 & 13,13 \\
\hline Relato & 2 & - & 1 & 14 & 17 & 5,07 \\
\hline Revisão & 2 & 2 & - & 36 & 40 & 11,94 \\
\hline Total & 23 & 5 & 1 & 306 & 335 & 100 \\
\hline
\end{tabular}

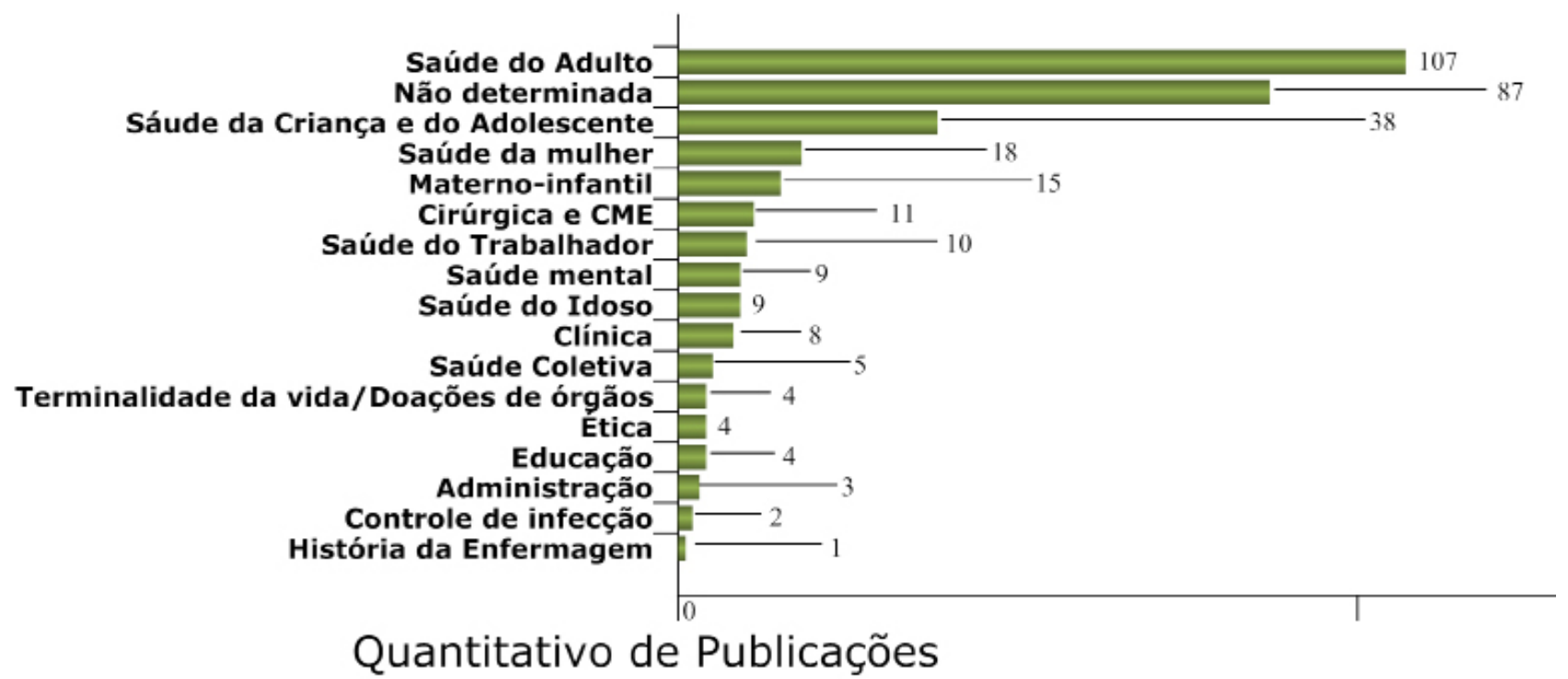

Gráfico 1- Temáticas abordadas nos estudos sobre o cuidado de enfermagem. Curitiba, 2011 
Tabela 2 - Atributos do cuidado de enfermagem por base de dados. Curitiba, 2011

\begin{tabular}{|c|c|c|c|c|c|c|}
\hline \multirow{2}{*}{$\begin{array}{c}\text { ATRIBUTOS DO } \\
\text { CUIDADO }\end{array}$} & \multicolumn{4}{|c|}{ BASE DE DADOS } & \multicolumn{2}{|c|}{ Total } \\
\hline & BDENF & LILACS & MEDLINE & SciELO & $\mathbf{n}$ & $(\%)$ \\
\hline $\begin{array}{l}\text { Importância do } \\
\text { conhecimento }\end{array}$ & 5 & 2 & - & 59 & 66 & 17,19 \\
\hline $\begin{array}{l}\text { Sistematização do } \\
\text { cuidado }\end{array}$ & 4 & - & - & 42 & 46 & 11,98 \\
\hline $\begin{array}{l}\text { Complexidade do } \\
\text { cuidado }\end{array}$ & 1 & 2 & - & 42 & 45 & 11,72 \\
\hline $\begin{array}{l}\text { Cuidado } \\
\text { individualizado }\end{array}$ & 1 & - & - & 42 & 43 & 11,2 \\
\hline $\begin{array}{l}\text { Utilização de } \\
\text { tecnologias }\end{array}$ & 1 & - & 1 & 29 & 31 & 8,07 \\
\hline $\begin{array}{l}\text { Cuidado } \\
\text { humanizado }\end{array}$ & 2 & - & - & 22 & 24 & 6,25 \\
\hline Educação em saúde & 1 & 1 & - & 16 & 18 & 4,69 \\
\hline $\begin{array}{l}\text { Interação enfermeiro- } \\
\text { paciente }\end{array}$ & 2 & - & - & 15 & 17 & 4,43 \\
\hline Cuidado cultural & 3 & - & - & 13 & 16 & 4,17 \\
\hline $\begin{array}{l}\text { Cuidado de si; } \\
\text { Autocuidado }\end{array}$ & 3 & - & - & 11 & 14 & 3,65 \\
\hline $\begin{array}{l}\text { Cuidado } \\
\text { especializado }\end{array}$ & - & - & - & 14 & 14 & 3,65 \\
\hline $\begin{array}{l}\text { Organização do } \\
\text { cuidado }\end{array}$ & 2 & - & - & 8 & 10 & 2,60 \\
\hline $\begin{array}{l}\text { Importância da } \\
\text { comunicação }\end{array}$ & 1 & - & - & 8 & 9 & 2,34 \\
\hline Cuidado ético & - & - & - & 7 & 7 & 1,82 \\
\hline $\begin{array}{l}\text { Pratica baseada em } \\
\text { evidências }\end{array}$ & - & - & - & 6 & 6 & 1,56 \\
\hline Cuidado integral & - & - & - & 4 & 4 & 1,04 \\
\hline $\begin{array}{l}\text { Cuidado } \\
\text { inespecífico }\end{array}$ & - & - & - & 4 & 4 & 1,04 \\
\hline $\begin{array}{l}\text { Controle de } \\
\text { infecção }\end{array}$ & - & - & - & 2 & 2 & 0,52 \\
\hline Lúdico & - & - & - & 2 & 2 & 0,52 \\
\hline $\begin{array}{l}\text { Prática social } \\
\text { empreendedora }\end{array}$ & - & - & - & 2 & 2 & 0,52 \\
\hline Avaliação em saúde & - & - & - & 1 & 1 & 0,26 \\
\hline $\begin{array}{l}\text { História da } \\
\text { enfermagem }\end{array}$ & - & - & - & 1 & 1 & 0,26 \\
\hline $\begin{array}{l}\text { Processo ensino } \\
\text { aprendizagem }\end{array}$ & - & - & - & 1 & 1 & 0,26 \\
\hline Relações de poder & - & - & - & 1 & 1 & 0,26 \\
\hline Total & 26 & 5 & 1 & 352 & 384* & 100 \\
\hline
\end{tabular}

Legenda: *O total ultrapassa o número de artigos devido a uma mesma publicação possuir mais de um atributo do cuidado. 


\section{DISCUSSÃO}

Ressalta-se na tabela 1 a prevalência de estudos qualitativos, o que remete a um modo de cuidar baseado não apenas nas percepções, mas também na consideração do contexto dos indivíduos assistidos, e aponta indícios da desvinculação do modelo biomédico, tão arraigado na profissão até o século passado. Investigação apontou a tendência de pesquisadores em Enfermagem para os estudos qualitativos, o que ressalta a valorização da compreensão e interpretação de fenômenos, além da consideração dos significados que os outros atribuem a suas práticas ${ }^{(6)}$.

Acredita-se que o elevado número de estudos voltados para a saúde do adulto (Gráfico 1) ocorreu devido ao aumento da espectativa de vida, o que leva à preocupação com a saúde dos indivíduos nessa fase, visto que os problemas de saúde enfrentados pelos idosos não surgem aos 60 anos; resultam, muitas vezes, de experiências anteriores, como condições de moradia, educação, alimentação, higiene e prática de atividades físicas ${ }^{(7)}$.

A seguir a discussão das três categorias identificadas.

\section{Importância do conhecimento para a efetivação do cuidado}

Conforme tabela 2, prevaleceu $(\mathrm{n}=66 ; 17,19 \%)$ o atributo correspondente à importância do conhecimento para a efetivação do cuidado. Compartilha-se a afirmação de que o enfermeiro, para prestar a assistência de qualidade, deve estar alicerçado no conhecimento, permeado pela atualização e capacitação constante ${ }^{(8)}$. Revisão da literatura, cujo objetivo foi evidenciar o estado da arte da temática família da criança com anemia falciforme, demonstrou a necessidade de conhecimento científico sobre a doença para a assistência de enfermagem ${ }^{(9)}$.

No que concerne ao conhecimento do enfermeiro, aponta-se artigo extraído de uma tese de doutorado, cujo cenário de pesquisa foi o campo de regulação da fecundidade em um serviço público de saúde ${ }^{(10)}$. As autoras ressaltam a interferência do poder institucional, representado pelo poder médico, como fator limitador da atuação das enfermeiras. Afirmam ainda que o conhecimento torna-se, nesse sentido, uma ferramenta importante para a mudança dessa realidade.

Outros autores colocam que o enfermeiro se fortalece enquanto membro da equipe multidisciplinar por meio do conhecimento ${ }^{(11)}$. Nessa perspectiva, ressalta-se, no presente estudo, a relevância desse atributo no que condiz à identidade do cuidado de enfermagem, presente nas publicações da primeira década do século XXI.

É inquestionável a necessidade dos profissionais que prestam cuidados adquirirem conhecimento, no entanto, explora-se no presente estudo a importância do conhecimento de acordo com uma segunda vertente, a do (des)conhecimento do ser cuidado. Para tanto, aponta-se um estudo etnográfico, cujo objetivo foi identificar a percepção de risco de infecção pelo HIV de mulheres moradoras em uma favela localizada na cidade de São Paulo, Brasil. Os resultados evidenciaram que as mulheres possuem conhecimento sobre a doença, sua forma de transmissão e de como prevení-la, no entanto, não percebem o risco de adquirir o vírus da imunodeficiência humana ${ }^{(12)}$.

Nessa perspectiva, ressalta-se estudo qualitativo descritivo, cujo objetivo foi identificar a experiência vivida pelos familiares de crianças internadas em Unidade de Terapia Intensiva Neonatal em um hospital de Curitiba-Paraná ${ }^{(13)}$. Os resultados evidenciaram que, apesar do atendimento prestado pela equipe multiprofissional, os familiares apresentam a necessidade em obter mais informações, orientação, aconselhamentos e apoio. Esses dados fortalecem o (des)conhecimento do ser humano cuidado e de seus familiares, o que evidencia a importância do conhecimento para a efetividade do cuidado de enfermagem.

\section{Sistematização do cuidado}

A prática de um cuidado sistematizado foi evidenciada em muitos dos resumos analisados, a citar o estudo de revisão bibliográfica cujo objetivo foi analisar como tem ocorrido a implantação da Sistematização da Assistência de Enfermagem (SAE) no serviço de saúde hospitalar do Brasil, no período de 1986 a $2005^{(14)}$. As autoras destacam que a finalidade da implementação é organizar o cuidado a partir da adoção de um método sistemático, proporcionando ao enfermeiro a (re)definição da sua ação. Para fins de categorização, considerou-se, no presente estudo, a sistematização da assistência sinônimo de cuidado sistematizado.

Ressalta-se, ainda, estudo qualitativo que teve por finalidade compreender o significado da SAE para os profissionais de saúde. As autoras afirmam que o enfermeiro que sistematiza sua prática adquire mais autonomia, bem como possibilita uma melhor aproximação/interação entre equipe, usuário e demais profissionais ${ }^{(15)}$. Ao utilizar a SAE, o enfermeiro proporciona melhora na qualidade da assistência prestada ${ }^{(16)}$. 
Como uma maneira de sistematizar a assistência de enfermagem, a utilização de um Processo de Enfermagem é apontado como uma tentativa relevante em universalizar a linguagem entre a categoria profissional, além de possibilitar a excelência no cuidado prestado ${ }^{(17)}$. Nesse sentido, reafirma-se a sistematização e a organização como integrantes da identidade do cuidado de enfermagem presente nas publicações do início deste século.

Assinala-se a utilização de tecnologias como integrante dessa identidade do cuidado de enfermagem na primeira década do século XXI. As tecnologias encontradas nas publicações são as mais diversas, e incluem desde o acolhimento, considerado como tecnologia leve por alguns autores ${ }^{(18)}$, até as tecnologias avançadas, que abarcam as máquinas e equipamentos que oferecem suporte de vida aos pacientes, bem como dispositivos considerados tecnologia de ponta.

Em investigação qualitativa desenvolvida em uma Unidade de Terapia Intensiva de um hospital de Santa Catarina, emergiu dos resultados o predomínio de tecnologias pouco centradas nas relações entre os sujeitos. Os autores afirmam que, diante dos resultados, as tecnologias afastam os profissionais de uma convivência interpessoal ${ }^{(19)}$. Em contrapartida, cita-se uma reflexão, construída no sentido de trabalhar com a temática humanização em terapia intensiva. Nela os autores afirmam que a utilização das tecnologias é, por vezes, interpretada como cuidado desumanizado, no entanto, é por meio desta que, muitas vezes, se possibilita manter o paciente vivo $^{(20)}$.

Nesse sentido, compartilha-se da opinião dos autores, ao afirmarem que os enfermeiros não devem limitar as ações de cuidar, mesmo com a existência da tecnologia entre o profissional e o paciente ${ }^{(20)}$. Para tanto, aponta-se a importância na utilização das tecnologias como ferramentas de apoio à assistência de um cuidado sistematizado e organizado, de maneira que o foco do enfermeiro seja sempre o cuidado humanizado.

\section{Cuidado humanizado pautado nas relações e inte- rações}

Nessa categoria estão incluídos os seguintes atributos identificados nos estudos: complexidade do cuidado, cuidado individualizado, cuidado humanizado, interação enfermeiro-paciente, cuidado ético, cuidado integral (Tabela 2). Esses atributos representam 42,7\% das produções científicas analisadas. Com base no pensamento complexo busca-se o entendimento da complexidade do cuidado, na qual a prática da enfer- magem vai além dos procedimentos técnicos, e também extrapola as definições abstratas, incluindo as vivências e percepções. Desta forma, o cuidar complexo abrange

cuidar do outro, cuidar do eu, perceber, se preocupar, estar com o outro, estar para ouvir, ver, experimentar e conhecer, é postura ética e estética em relação ao mundo, [...] é refletir, alimentar, tocar, aliviar a dor, ouvir, hidratar, medicar, preparar para curativos, cirurgias, exames, preparar para a vida e para a morte ${ }^{(21: 688)}$.

Nesse sentido as práticas de cuidado complexo consideram as relações e interações do ser humano com o ambiente e com outros seres humanos, dentre os quais os membros de sua família e a equipe que presta cuidados a sua saúde ${ }^{(21)}$. Assim, procura-se oferecer um cuidado pautado na subjetividade e interação, de maneira que exista troca de conhecimentos, experiências e vivências negativas e positivas.

O cuidado no qual se evidencia a interação com o paciente ancora-se nos pressupostos de que o enfermeiro possa, por meio das percepções, presença, compromisso, solidariedade e interações humanas, auxiliar o paciente a reorganizar sua existência ao passar pela experiência da doença ${ }^{(22)}$. Nessa perspectiva, a identidade do cuidado abarca mais que uma boa ação, envolve momentos de atenção, zelo, responsabilidade e de envolvimento afetivo com o outro. Manifesta-se na preservação do potencial saudável dos cidadãos e depende de uma concepção ética que contemple a vida como um bem valioso em si. A essência do cuidado está presente em toda a vida do ser humano ${ }^{(23)}$.

\section{CONSIDERAÇÕES FINAIS}

A inquietação em determinar a identidade do cuidado de enfermagem surge desde os primórdios da profissão e se estende aos dias de hoje, pois, a evolução científica exige atualizações constantes e ressalta a necessidade de definir as especificidades profissionais. É conhecida, desde a formação inicial, a essência da enfermagem centrada no cuidado ao ser humano e suas coletividades. No entanto, busca-se aperfeiçoar o entendimento de quais os atributos e especificidades que diferenciam o cuidado humano do cuidado profissional.

Esta revisão possibilitou observar que a identidade do cuidado de enfermagem na primeira década do século XXI pautou-se no conhecimento do profissional enfermeiro, na sistematização da assistência de enfermagem e na humanização do cuidado. $O$ cuidado 
amplia-se do fazer individual-coletivo para uma visão complexa do ser e estar cuidado-cuidando. Espera-se que os aspectos apontados acerca da identidade do cuidado, ao evidenciar quais são os atributos do cuidado identificados, possam contribuir para reflexões futuras da prática profissional da enfermagem.

\section{REFERENCIAS}

1. Heidegger M. Ser e tempo. Parte I. $15^{\mathrm{a}}$ ed. RJ: Vozes; 2005.

2. Ayres JRCM. O cuidador, os modos de ser (do) humano e as práticas de saúde. Saude soc. 2004;13(3):16-29.

3. Abbagnano N. Dicionário de filosofia. $5^{\mathrm{a}}$ ed. São Paulo: Martins Fontes; 2007.

4. Alves MDS, Oriá MOB, Franco ES, Costa MS, Barroso MGT. História da enfermagem registrada nas artes plásticas: do século XVI ao século XX. Texto Contexto Enferm. 2005;14(4):513-9.

5. Ganong LH. Integrative reviews of nursing research. Res. nurs. health 1987;10(1):1-11.

6. Cruz ATCT, Dodt RCM, Oriá MOB, Alves MDS. Enfermagem em Unidade de Terapia Intensiva Neonatal: perfil da produção científica brasileira. Cogitare enferm. 2011;16(1):141-7.

7. Camargos MCS, Rodrigues RN, Machado CJ. Expectativa de vida saudável para idosos brasileiros, 2003. Ciênc. saúde colet. 2009;14(5):1903-09.

8. Faustino AM, Reis PED, Kamada I, Jesus CAC, Izidorio SR, Ferreira SS. O conhecimento de enfermeiros acerca dos novos descritores de classificação para úlcera por pressão: estudo descritivo. Online Braz J Nurs. [Internet] 2010;9(1) [acesso em 30 jun 2011]. Disponível: http://www.objnursing.uff.br/ index.php/nursing/article/view/j.1676-4285.2010.2800/629

9. Rodrigues CCM, Araújo IEM, Melo LL. A família da criança com doença falciforme e a equipe enfermagem: revisão crítica. Rev. Bras. Hematol. Hemoter. 2010;32(3):257-64.

10. Coelho EAC, Fonseca RMGS. Atuação de enfermeiras no campo da regulação da fecundidade em um serviço público de saúde. Rev Esc Enferm USP. 2004;38(1):37-45.

11. Castanha ML, Zagonel IPS. A prática do cuidar do ser enfermeiro sob o olhar da equipe de saúde. Rev. bras. enferm. 2005;58(5):556-62.
12. Praça NS, Gualda DMR. Risco de infecção pelo HIV: como mulheres moradoras em uma favela se percebem na cadeia de transmissão do vírus. Rev. Latino-Am. Enfermagem 2003;11(1):14-20

13. Centa ML, Moreira EC, Pinto MNGHR. A experiência vivida pelas famílias de crianças hospitalizadas em uma unidade de terapia intensiva neonatal. Texto Contexto Enferm. 2004;13(3):444-51.

14. Castilho NC, Ribeiro PC, Chirelli MQ. A implementação da sistematização da assistência de enfermagem no serviço de saúde hospitalar do Brasil. Texto Contexto Enferm. 2009;18(2):280-9.

15. Nascimento KC, Backes DS, Koerich MS, Erdmann AL. Sistematização da assistência de enfermagem: vislumbrando um cuidado interativo, complementar e multiprofissional. RevEsc Enferm USP. 2008;42(4):643-8.

16. Lima GS, Cavalcante TMC, Isabella APJ, Magalhães AS. Assistência de enfermagem a um paciente infartado portador de HIV, baseada na teoria do autocuidado: estudo de caso. Acta Paul. Enferm. 2007;20(4):452-7.

17. Freitas MC, Mendes MMR. Idoso vítima de queimaduras: identificação do diagnóstico e proposta de intervenção de enfermagem. Rev. bras. enferm. 2006;59(3):362-6.

18. Rossi FR, Lima MADS. Acolhimento: tecnologia leve nos processos gerenciais do enfermeiro. Rev. bras. enferm. 2005;58(3):305-10.

19. Pinho LB, Santos SMA, Kantorski LP. Análise do processo de trabalho da enfermagem na unidade de terapia intensiva. Texto Contexto Enferm. 2007;16(4):703-11.

20. Silva RCL, Porto IS, Figueiredo NMA. Reflexões acerca da assistência de enfermagem e o discurso de humanização em terapia intensiva. Esc. Anna Nery. 2008;12(1):156-9.

21. Silva AL, Freitas MG. O ensino do cuidar na Graduação em Enfermagem sob a perspectiva da complexidade. Rev Esc Enferm USP. 2010;44(3):687-93.

22. Cunha PJ, Zagonel IPS. As relações interpessoais nas ações de cuidar em ambiente tecnológico hospitalar. Acta Paul. Enferm. 2008;21(3):412-9.

23. FonsecaAM,CamposACV,CottaFMP,BorelliLR,DutraBS, Santana JCB. Reflexões éticas sobre o cuidado humanizado na percepção dos enfermeiros. Cienc. saude 2010;3(1):2-8. 